\title{
INITIAL AND BOUNDARY VALUE PROBLEMS FOR PARTIAL FUNCTIONAL DIFFERENTIAL EQUATIONS
}

\author{
S.K. NTOUYAS and P.CH. TSAMATOS \\ University of Ioannina, Department of Mathematics \\ 45110 Ioannina, Greece
}

(Received June, 1994; Revised August, 1996)

\begin{abstract}
In this paper we study the existence of solutions to initial and boundary value problems of partial functional differential equations via a fixed-point analysis approach. Using the topological transversality theorem we derive conditions under which an initial or a boundary value problem has a solution.

Key words: Partial Functional Differential Equations, Initial Value Problems, Boundary Value Problems, Topological Transversality, A Priori Bounds.
\end{abstract}

AMS subject classifications: $35 \mathrm{R} 10$.

\section{Introduction}

In this paper we study initial and boundary value problems for a class of semilinear, abstract functional differential equations. This class is characterized by the fact that the associated homogeneous, linear differential equation generates a strongly continuous linear evolution system of compact operators.

More precisely, we consider the nonlinear Volterra integral equation

$$
x(t)=W(t,) x(0)+\int_{0}^{t} W(t, s) f\left(s, x_{s}\right) d s, t \in[0, b]
$$

together with an initial condition

$$
x_{0}=\varphi
$$

or with a linear boundary condition

$$
L x=\alpha .
$$

Our notations follow that of Hale [5] and Travis and Webb [12]. $X$ denotes a Banach space. $C=C([-r, 0], X)$ is the Banach space of continuous $X$-valued functions with 
supremum norm $\|\cdot\|$, i.e,. $\|\phi\|=\sup \{|\phi(\theta)|:-r \leq \theta \leq 0\}$. If $u$ is a continuous function from an interval $[-r, b], b>0$ to $X$, then for $t \in[0, b], u_{t}$ is the element of $C$ given by $u_{t}(\theta)=u(t+\theta), \theta \in[-r, 0] . f:[0, b] \times C \rightarrow X$ is a function. Also $\{W(t, s)$ : $0 \leq s \leq t \leq b\}$ is a strongly continuous family of evolution operators on $X$. Finally, $L$ is a bounded linear operator from $C([-r, b], X)$ into $X$ and $\alpha \in X$ is given.

If the evolution system $W(t, s)$ is generated by a family of densely defined linear operators $A(t)$, then equation (1.1) is an integral form of the abstract functional differential equation

$$
\begin{gathered}
x^{\prime}(t)=A(t) x(t)+f\left(t, x_{t}\right), 0 \leq t \leq b \\
x_{0}=\phi .
\end{gathered}
$$

Initial value problems for partial functional differential equations have been studied by Travis and Webb [12, 13], Fitzgibbon [2] and Rankin [11].

When $r=0$, i.e., in the case of ordinary differential equations, the boundary value problem (1.1)-(1.2) has been studied by Ward [15]. Also, Rankin [10] considered boundary value problems for partial functional differential equations, with $A(t)=$ $A$, independent of $t$, the infinitesimal generator of a strongly continuous semigroup $T(t)$ and the boundary condition (1.3) in the case where $L$ has the more specific form $L x=M x_{0}+N x_{b}$, with $M$ and $N$ bounded linear operators from $C$ into $C$.

In this paper we prove existence results for initial and boundary value problems by using the "Topological Transversality Method" of Granas [1]. This method reduces the problem of the existence of solutions of an initial or boundary value problem to the establishment of suitable a priori bounds for solutions of these problems.

The advantage of this method for initial value problems (at least in the case of ordinary differential equations) is that this yields simultaneously the existence of solutions and the maximal interval of existence.

Many recent papers deal with this method and the interested reader is referred to $[4,7,9,14]$ and the references given therein.

As a model for the class of partial functional differential equations we study, one may take the equation

$$
\begin{gathered}
w_{t}(x, t)=w_{x x}(x, t)+f(t, w(x, t-r)), 0 \leq x \leq b, t \geq 0 \\
w(0, t)=w(b, t)=0, t \geq 0 \\
w(x, t)=\phi(x, t), 0 \leq x \leq b,-r \leq t \leq 0,
\end{gathered}
$$

(see $[2,11-13]$.

The paper is organized as follows: In Section 2, we present some preliminaries and facts about the evolution system $W(t, s)$ and the linear operator $L$. In Section 3 we consider initial value problems and we extend the above-mentioned method of Topological Transversality of Granas to the partial functional differential equations. We prove the basic existence theorem (Theorem 3.1) by assuming a priori bounds on solutions. It is well known (see e.g., [16]) that only the continuity of $f$ is not sufficient to assure local existence of solutions, even when $X$ is a Hilbert space. Therefore, one has to restrict either the function $f$ or the evolution system. In most of the previous works further restrictions on $f$ are imposed. The function $f$ is as- 
sumed to be locally Lipschitz or monotone or completely continuous. Fitzgibbon [2] assumed that the function $f$ is continuous while the evolution operator compact. Here we assume, following Ward [14], that the function $f$ satisfies Caratheodory-type conditions, which does not imply that $f$ is completely continuous, and that the evolution operator is compact. In order to apply this basic existence theorem to obtain global existence of solutions, we establish the desired a priori bounds for solutions in Theorem 3.2.

In Section 4 we study boundary value problems. Finally, an application of a partial functional differential equation of Sobolev type, is given in Section 5 .

\section{Preliminaries}

Throughout this paper we shall make the following assumptions on the evolution system $W(t, s)$ :

$\left(W_{1}\right) W(t, s) \in L(X)$, the space of bounded linear transformations on $X$, whenever $0 \leq s \leq t \leq b$ and for each $x \in X$ the mapping $(t, s) \rightarrow W(t, s) x$ is continuous.

$\left(W_{2}\right) W(t, s) W(s, r)=W(t, r), 0 \leq r \leq s \leq t \leq b$.

$\left(W_{3}\right) W(t, t)=I$, the identity operator on $X$.

$\left(W_{4}\right) W(t, s)$ is a compact operator whenever $t-s>0$.

Sufficient conditions for $\left(W_{1}\right)-\left(W_{4}\right)$ to hold may be found in Friedman [3]. In what follows, $W(t, 0)$ is extended to $[-r, 0]$ by the identity operator.

The operator $L$ in $(3.1)$ is a continuous linear operator from $C([-r, b], X)$ into $X$. We define $\widetilde{L} \in L(X)$ by

$$
\widetilde{L} x=L[W(\cdot, 0) x]
$$

for all $x \in X$. We will assume in the sequel that the operator $\widetilde{L}$ has a bounded inverse $\widetilde{L}^{-1}$.

Before stating our basic existence theorems we need the following lemma which is referred to as the "Leray-Schauder alternative", [1, p. 61].

Lemma 2.1: Let $B$ be a convex subset of a normed linear space $E$ and assume $0 \in B$. Let $F: B \rightarrow B$ be a completely continuous operator, i.e., it is continuous and the image of any bounded set is included in a compact set, and let

$$
\mathcal{E}(F)=\{x \in B: x=\lambda F x \text { for some } 0<\lambda<1\}
$$

Then either $\&(F)$ is unbounded or $F$ has a fixed point.

\section{Initial Value Problems}

In this section we give our basic existence result for the initial value problem (IVP) (1.1)-(1.2). We assume, following Ward [15], that the function $f$ satisfies the following Caratheodory-type conditions:

$\left(C_{1}\right)$ For each $t \in[0, b]$, the function $f(t, \cdot): C \rightarrow X$ is continuous, and for each $x \in C$, the function $f(\cdot, x):[0, b] \rightarrow X$ is strongly measurable.

$\left(C_{2}\right)$ For every positive integer $k$ there exists $g_{k} \in L^{1}([0, b])$ such that for a.a. 
$t \in[0, b]$

$$
\sup _{\|x\| \leq k}|f(t, x)| \leq g_{k}(t) .
$$

Theorem 3.1: Let $\{W(t, s): 0 \leq s \leq t \leq b\}$ satisfy $\left(W_{1}\right)-\left(W_{4}\right)$ and $f:[0, b] \times C \rightarrow X$ a function satisfying $\left(C_{1}\right)$ and $\left(C_{2}\right)$.

Assume that there exists a constant $K$ such that

$$
\|x\|_{b} \equiv \sup _{-r \leq t \leq b}|x(t)| \leq K
$$

for each solution $x$ of

$$
\begin{gathered}
x(t)=\lambda W(t, 0) x(0)+\lambda \int_{0}^{t} W(t, s) f\left(s, x_{s}\right) d s, t \in[0, b] \\
x_{0}=\phi
\end{gathered}
$$

for any $\lambda \in(0,1)$.

Then the IVP (1.1)-(1.2) has at least one solution on $[-r, b]$.

Proof: We will rewrite $(1.1)$ as follows. For $\phi \in C$ define $\tilde{\phi} \in C_{b}, C_{b}=$ $C([-r, b], X)$ by

$$
\tilde{\phi}(t)=\left\{\begin{array}{cc}
\phi(t), & -r \leq t \leq 0 \\
W(t, 0) \phi(0), & 0<t \leq b .
\end{array}\right.
$$

If $x(t)=y(t)+\widetilde{\phi}(t), t \in[-r, b]$ it is easy to see that $y$ satisfies

$$
\begin{gathered}
y_{0}=0 \\
y(t)=\int_{0}^{t} W(t, s) f\left(s, y_{s}+\widetilde{\phi}_{s}\right) d s
\end{gathered}
$$

if and only if $x$ satisfies (1.1) and $x_{0}=\phi$.

Define $F: C_{b}^{0} \rightarrow C_{b}^{0}, C_{b}^{0}=\left\{y \in C_{b}: y_{0}=0\right\}$ by

$$
(F y)(t)=\left\{\begin{array}{cc}
0, & -r \leq t \leq 0 \\
\int_{0}^{t} W(t, s) f\left(s, y_{s}+\tilde{\phi}_{s}\right) d s, & 0 \leq t \leq b .
\end{array}\right.
$$

It will now be shown that $F$ is a completely continuous operator.

Let $B_{k}=\left\{y \in C_{b}^{0}:\|y\| \leq k\right\}$ for some $k \geq 1$. We first show that $F$ maps $B_{k}$ into an equicontinuous family. Let $y \in B_{k}$ and $t_{1}, t_{2} \in[0, b]$ and $\epsilon>0$. Then if $0<\epsilon<t_{1}<t_{2} \leq b$

$$
\begin{aligned}
& \left|(F y)\left(t_{1}\right)-(F y)\left(t_{2}\right)\right|= \\
& \left|\int_{0}^{t_{1}} W\left(t_{1}, s\right) f\left(s, y_{s}+\tilde{\phi}_{s}\right) d s-\int_{0}^{t_{2}} W\left(t_{2}, s\right) f\left(s, y_{s}+\tilde{\phi}_{s}\right) d s\right|
\end{aligned}
$$




$$
\begin{aligned}
& \leq\left|\int_{0}^{t_{1}-\epsilon}\left[W\left(t_{1}, s\right)-W\left(t_{2}, s\right)\right] f\left(s, y_{s}+\tilde{\phi}_{s}\right) d s\right| \\
& +\left|\int_{t_{1}-\epsilon}^{t_{1}}\left[W\left(t_{1}, s\right)-W\left(t_{2}, s\right)\right] f\left(s, y_{s}+\tilde{\phi}_{s}\right) d s\right| \\
& +\left|\int_{t_{1}}^{t_{2}} W\left(t_{2}, s\right) f\left(s, y_{s}+\tilde{\phi}_{s}\right) d s\right| \\
& \leq \int_{0}^{t_{1}-\epsilon}\left|W\left(t_{1}, s\right)-W\left(t_{2}, s\right)\right| g_{k^{\prime}}(s) d s \\
& +2 N \int_{t_{1}-\epsilon}^{t_{1}} g_{k^{\prime}}(s) d s+N \int_{t_{1}}^{t_{2}} g_{k^{\prime}}(s) d s \\
& k^{\prime}=k+\|\tilde{\phi}\| \\
& N=\sup \{|W(t, s)|: 0 \leq s \leq t \leq b\} .
\end{aligned}
$$

We remark that $N$ is finite, by the principle of uniform boundedness, since $W(t, s)$ is strongly continuous on the compact triangle $0 \leq s \leq t \leq b$.

The right-hand side of (3.2) is independent of $y \in B_{k}$ and tends to zero as $t_{2}-$ $t_{1} \rightarrow 0$ and $\epsilon$ sufficiently small, since the fact of compactness of $W(t, s)$ for $t-s>0$, implies the continuity of $W(t, s)$ in the operator norm.

Thus $F$ maps $B_{k}$ into an equicontinuous family of functions. It is easy to see that the family $B_{k}$ is uniform bounded.

Notice that we considered here only the case $0<t_{1}<t_{2}$, since the other cases $t_{1}<t_{2}<0$ or $t_{1}<0<t_{2}$ are very simple.

Since we have shown $F B_{k}$ is an equicontinuous collection, it suffices by the Arzela-Ascoli theorem to show that $F$ maps $B_{k}$ into a precompact set in $X$. define

Let $0<t \leq b$ be fixed and $\epsilon$ a real number satisfying $0<\epsilon<t$. For $y \in B_{k}$ we

$$
\begin{gathered}
\left(F_{\epsilon} y\right)(t)=\int_{0}^{t-\epsilon} W(t, s) f\left(s, y_{s}+\tilde{\phi}_{s}\right) d s \\
=W(t, t-\epsilon) \int_{0}^{t-\epsilon} W(t-\epsilon, s) f\left(s, y_{s}+\tilde{\phi}_{s}\right) d s .
\end{gathered}
$$


Since $W(t, t-\epsilon)$ is a compact operator, the set $Y_{\epsilon}(t)=\left\{\left(F_{\epsilon} y\right)(t): y \in B_{k}\right\}$ is precompact in $X$, for every $\epsilon, 0<\epsilon<t$. Moreover, for every $y \in B_{k}$ we have

$$
\begin{aligned}
\left|(F y)(t)-\left(F_{\epsilon} y\right)(t)\right| & \leq \int_{t-\epsilon}^{t}\left|W(t, s) f\left(s, y_{s}+\tilde{\phi}_{s}\right)\right| d s \\
& \leq N \int_{t-\epsilon}^{t} g_{k}(s) d s
\end{aligned}
$$

Therefore, there are precompact sets arbitrary close to the set $\left\{(F y)(t): y \in B_{k}\right\}$. Hence, the set $\left[(F y)(t): y \in B_{k}\right\}$ is precompact in $X$.

It remains to show that $F: C_{b}^{0} \rightarrow C_{b}^{0}$ is continuous. Let $\left\{u_{n}\right\}_{0}^{\infty} \subseteq C_{b}^{0}$ with $u_{n} \rightarrow u$ in $C_{b}^{0}$. Then there is an integer $M$ such that $\left|u_{n}(t)\right| \leq M$ for all $n$ and $t \in[0, b]$, so $u_{n} \in B_{M}$ and $u \in B_{M \cdot \sim}$ By $\left(C_{1}\right) f\left(t, u_{n}(t)+\tilde{\phi}_{s}\right) \rightarrow f\left(t, u(t)+\tilde{\phi}_{s}\right)$ for each $t \in[0, b]$, and since $\left|f\left(t, u_{n}(t)+\tilde{\phi}_{s}\right)-f\left(t, u(t)+\tilde{\phi}_{s}\right)\right| \leq 2 g_{M^{\prime}}(t), M^{\prime}=M+\|\phi\|$ we have by dominated convergence

$$
\begin{gathered}
\left\|F u_{n}-F u\right\|=\sup _{t \in[0, b]}\left|\int_{0}^{t} W(t, s)\left[f\left(s, u_{n}(s)+\tilde{\phi}_{s}\right)-f\left(s, u(s)+\tilde{\phi}_{s}\right)\right] d s\right| \\
\leq N \int_{0}^{b}\left|f\left(s, u_{n}(s)+\tilde{\phi}_{s}\right)-f\left(s, u(s)+\tilde{\phi}_{s}\right)\right| d s \rightarrow 0 .
\end{gathered}
$$

Thus $F$ is continuous. This completes the proof that $F$ is completely continuous.

Finally, we shall prove that the set $\&(F)=\left\{y \in C_{b}^{0}: y \in \lambda F y, \lambda \in(0,1)\right\}$ is bounded. Indeed, for every solution $y$ in $C_{b}^{0}$ of (3.1) the function $x=y+\widetilde{\varphi}$ is a solution of $(1.1)_{\lambda}$. Thus, by hypothesis we have

$$
\|y\|_{b} \leq K+\|\tilde{\varphi}\| \text {. }
$$

Consequently, by Lemma 2.1 , the operator $F$ has a fixed point $y^{*}$ in $C_{b}^{0}$. Then $x^{*}=$ $y^{*}+\widetilde{\phi}$ is a solution of the initial value problem (1.1)-(1.2). The proof of the theorem is now complete.

The applicability of Theorem 3.1 depends upon the existence of a priori bounds for the solutions of the IVP $(1.1)_{\lambda^{-}}(1.2)$, which are independent of $\lambda, \lambda \in(0,1)$.

In the next theorem we give conditions under which a solution of the IVP (1.1)(1.2) exists on the interval $[-r, b]$.

Theorem 3.2: Let $\{W(t, s): 0 \leq s \leq t \leq b\}$ satisfy $\left(W_{1}\right)-\left(W_{4}\right)$ and $f:[0, b] \times C \rightarrow X$ a function satisfying $\left(C_{1}\right)$ and $\left(C_{2}\right)$. Assume that:

$(H f)$ There exists an integrable function $m:[0, b] \rightarrow R^{+}$such that

$$
|f(t, \phi)| \leq m(t) \Omega(\|\phi\|), 0 \leq t \leq b, \phi \in C,
$$

where $\Omega:[0, \infty) \rightarrow(0, \infty)$ is a nondecreasing function with $\frac{1}{\Omega}$ locally integrable on $[0, \infty)$.

Then the IVP (1.1)-(1.2) has at least one solution on $[-r, b]$ provided that 


$$
N \int_{0}^{b} m(s) d s<\int_{\widehat{N}\|\phi\|}^{\infty} \frac{d s}{\Omega(s)}, \widehat{N}=\max \{1, N\} .
$$

where $N$ was defined in (3.3).

Proof: To prove the existence of a solution of the IVP (1.1)-(1.2) we apply Theorem 3.1. In order to apply this theorem we must establish the a priori bounds for the solutions of the IVP $(1.1)_{\lambda^{-}}(1.2), \lambda \in(0,1)$.

Let $x$ be a solution of the IVP $(1.1)_{\lambda^{-}}(1.2)$. Then we have

$$
|x(t)| \leq N\|\phi\|+N \int_{0}^{t} m(s) \Omega\left(\left\|x_{s}\right\|\right) d s, 0 \leq t \leq b .
$$

We consider the function $\ell$ given by

$$
\ell(t)=\sup \{|x(s)|:-r \leq s \leq t\}, \quad 0 \leq t \leq b .
$$

Let $t^{*} \in[-r, t]$ be such that $\ell(t)=\left|x\left(t^{*}\right)\right|$. If $t^{*} \in[0, t]$, by the previous inequality we have

$$
\begin{aligned}
\ell(t)= & \left|x\left(t^{*}\right)\right| \leq N\|\phi\|+N \int_{0}^{t^{*}} m(s) \Omega\left(\left\|x_{s}\right\|\right) d s \\
& \leq N\|\phi\|+N \int_{0}^{t} m(s) \Omega(\ell(s)) d s \\
& \leq \widehat{N}\|\phi\|+N \int_{0}^{t} m(s) \Omega(\ell(s)) d s, 0 \leq t \leq b .
\end{aligned}
$$

If $t^{*} \in[-r, 0]$ then $\ell(t)=\|\phi\|$ and the previous inequality obviously holds.

Denoting by $u(t)$ the right-hand side of the above inequality we have

$$
u(0)=\widehat{N}\|\phi\|, \ell(t) \leq u(t), 0 \leq t \leq b,
$$

and

$$
u^{\prime}(t)=N m(t) \Omega(\ell(t)) \leq N m(t) \Omega(u(t)), 0 \leq t \leq b .
$$

Then

$$
\frac{u^{\prime}(t)}{\Omega(u(t))} \leq N m(t), 0 \leq t \leq b
$$

or

$$
\int_{u(0)}^{u(t)} \frac{d s}{\Omega(s)} \leq N \int_{0}^{b} m(s) d s<\int_{u(0)}^{\infty} \frac{d s}{\Omega(s)}, 0 \leq t \leq b .
$$

This inequality implies that there is a constant $K$ such that $u(t) \leq K, t \in[0, b]$ and hence $\ell(t) \leq K, t \in[0, b]$. Since for every $t \in[0, b],\left\|x_{t}\right\| \leq \ell(t)$, we have 


$$
\|x\|_{b} \leq K
$$

where $K$ depends only on $b$ and the functions $m$ and $\Omega$. Hence our proof is complete $\square$

\section{Boundary Value Problems}

Consider in this section, the BVP (1.1)-(1.3).

Theorem 4.1: Let $\{W(t, s): 0 \leq s \leq t \leq b\}$ satisfy $\left(W_{1}\right)-\left(W_{4}\right)$ and $f:[0, b] \times C \rightarrow X$ a function satisfying $\left(C_{1}\right)$ and $\left(C_{2}\right)$. Assume that $L$ defined by $(2.1)$ has a bounded inverse $\widetilde{L}^{-1}$. Furthermore, assume that there exists a constant $K$ such that

$$
\|x\|_{b} \equiv \sup _{-r \leq t \leq b}|x(t)| \leq K
$$

for each solution $x$ of

$$
\begin{gathered}
x(t)=\lambda W(t, 0) x(0)+\lambda \int_{0}^{t} W(t, s) f\left(s, x_{s}\right) d s, t \in[0, b] \\
L x=\lambda \alpha
\end{gathered}
$$

for any $\lambda \in(0,1)$.

Then the BVP (1.1)-(1.3) has at least one solution on $[-r, b]$.

Proof: Following Kaminogo [6], define $S: C_{b} \rightarrow: C_{b},: C_{b}=C([-r, b], X)$ by

$$
S x=P x+Q x
$$

where

$$
P x=W(t, 0) \tilde{L}^{-1}(\alpha-L(Q x(0))), x \in C_{b}
$$

and

$$
(Q x)(t)=\left\{\begin{array}{cc}
0, & -r \leq t \leq 0 \\
\int_{0}^{t} W(t, s) f\left(s, x_{s}\right) d s, & 0 \leq t \leq b .
\end{array}\right.
$$

Since the mapping $x \rightarrow W(\cdot, 0) x$ is bounded and linear from $X$ into $C_{b}$ the operator $P$ is continuous and compact. The operator $Q$ is completely continuous as has been proved in Theorem 3.1. Also, the set associated with the operator $P$ is precompact because of the complete continuity of $Q$. Hence $S=P+Q$ is a completely continuous operator.

Also, the set $\&(S)=\left\{x \in C_{b}: x=\lambda S x, \lambda \in(0,1)\right\}$ is bounded by hypothesis. Thus, by Lemma 2.1, the operator $S$ has a fixed point $x^{*}$ in $C_{b}$. Hence $x^{*}$ is a solution of the BVP (1.1)-(1.3) and this completes the proof.

Theorem 4.2: Let $\{W(t, s): 0 \leq s \leq t \leq b\}$ satisfy $\left(W_{1}\right)-\left(W_{4}\right)$ and $f:[0, b] \times C \rightarrow X$ a function satisfying $\left(C_{1}\right),\left(C_{2}\right)$ and hypothesis $(H f)$ of Theorem 3.2 . Also assume that $\widetilde{L}$ defined by $(2.1)$ has a bounded inverse $\widetilde{L}^{-1}$.

Then if 


$$
N\left(N\left|\tilde{L}^{-1}\right||L|+1\right) \int_{0}^{b} m(s) d s<\int_{c}^{\infty} \frac{d s}{\Omega(s)}, c=N\left|\tilde{L}^{-1}\right||\alpha|
$$

the BVP (1.1)-(1.3) had at least one solution on $[-r, b]$.

Proof: We apply Theorem 4.1. In order to apply this theorem, we must establish the a priori bounds for the solutions of the BVP $(1.1)_{\lambda^{-}}(1.3)$. Let $x$ be a solution of the BVP $(1.1)_{\lambda^{-}}(1.3)$. Then

$$
x(t)=\lambda\left\{W(t, 0) \tilde{L}^{-1}(\alpha-L(Q x(0)))+(Q x)(t)\right\}
$$

with $Q$ defined by (4.3). From this we get

$$
\begin{aligned}
\mid x(t) \leq & N\left|\tilde{L}^{-1}\right|\left(|\alpha|+|L| N \int_{0}^{t} m(s) \Omega\left(\left\|x_{s}\right\|\right) d s\right)+N \int_{0}^{t} m(s) \Omega\left(\left\|x_{s}\right\|\right) d s \\
& \leq N\left|\tilde{L}^{-1}\right|\left(|\alpha|+N\left(N\left|\tilde{L}^{-1}\right||L|+1\right) \int_{0}^{t} m(s) \Omega\left(\left\|x_{s}\right\|\right) d s\right.
\end{aligned}
$$

or

$$
\ell(t) \leq N\left|\tilde{L}^{-1}\right|\left(|\alpha|+N\left(N\left|\tilde{L}^{-1}\right||L|+1\right) \int_{0}^{t} m(s) \Omega(\ell(s)) d s\right.
$$

with $\ell$ as defined in the proof of Theorem 3.2.

Denoting by $v(t)$ the right-hand side of the above inequality, we obtain

$$
\left.\int_{v(0)}^{v(s)} \frac{d s}{\Omega(s)} \leq N\left|\tilde{L}^{-1}\right| L \mid+1\right) \int_{0}^{b} m(s) d s<\int_{v(0)}^{\infty} \frac{d s}{\Omega(s)}, \quad 0 \leq t \leq b,
$$

from which we get

$$
\|x\|_{b} \leq K
$$

and the proof is now complete.

\section{An Application}

As an application, we consider the Cauchy problem for the partial functional differential equation of Sobolev type

$$
\begin{gathered}
(B x(t))^{\prime}+A x(t)=f\left(t, x_{t}\right), 0 \leq t \leq b \\
x(t)=\phi(t),-r \leq t \leq 0
\end{gathered}
$$

where $f$ satisfies $\left(C_{1}\right),\left(C_{2}\right)$ and $A$ and $B$ are closed linear operators with domains contained in a Banach space $X$ and ranges in a Banach space $Y, D(B) \subset D(A), B$ is bijective and $B^{-1}: Y \rightarrow D(B)$ is compact. This problem has been studied by J. Lightbourne III and S. Rankin III in [8] by considering the related integral equation 


$$
\begin{gathered}
y(t)=T(t) B \phi(0)+\int_{0}^{t} T(t-s) f\left(s, B^{-1} y_{s}\right) d s, 0 \leq t \leq b \\
y(t)=B \phi(t),-r \leq t \leq 0
\end{gathered}
$$

where $T(t), t \geq 0$ is the semigroup of bounded linear operators generated by $-A B^{-1}$, satisfying

$$
|T(t)| \leq M e^{\omega t}
$$

for some $M \geq 1$ and $\omega \in R$. It is obvious that if $x(t)$ solves (5.1)-(5.2) then $y(t)=$ $B x(t)$ solves (5.3)-(5.4).

By using the results of the previous sections we obtain the following result.

Theorem 5.1: Suppose that the above-mentioned conditions for $A$ and $B$ are satisfied and the function $f$ satisfies $\left(C_{1}\right),\left(C_{2}\right)$ and the condition $(H f)$. Moreover, assume that $T(t), t>0$ is compact and

$$
\left|B^{-1}\right| \int_{0}^{b} \widehat{m}(s) d s<\int_{c}^{\infty} \frac{d s}{s+\Omega(s)}
$$

where $\widehat{m}(t)=\max \left\{M m(t), \frac{\omega}{\left|B^{-1}\right|}\right\}$ and $c=\left|B^{-1}\right| M B\|\phi\|$. Then the problem (5.1)-(5.2) has at least one solution on $[-r, b]$.

Proof: We apply Theorem 3.1. Let $y$ be a solution of

$$
\begin{gathered}
y(t)=\lambda T(t) B \phi(0)+\lambda \int_{B \phi(t),-r \leq t \leq 0 .}^{t} T(t-s) f\left(s, B^{-1} y_{s}\right) d s, 0 \leq t \leq b \\
y(t)=B(t)
\end{gathered}
$$

Then we have

$$
|y(t)| \leq M e^{\omega t}|B|\|\phi\|+e^{\omega t} \int_{0}^{t} M e^{-\omega s} m(s) \Omega\left(\left|B^{-1}\right|\left\|y_{s}\right\|\right) d s
$$

or

$$
e^{-\omega t} \ell(t) \leq M|B|\|\phi\|+\int_{0}^{t} M e^{-\omega s} m(s) \Omega\left(\left|B^{-1}\right| \ell(s)\right) d s
$$

with $\ell$ as defined in the proof of Theorem 3.2.

Denoting by $u(t)$ the right-hand side of the above inequality we obtain

$$
u(0)=M|B|\|\phi\|, \quad \ell(t) \leq e^{w t} u(t), \quad 0 \leq t \leq b
$$

and

$$
\begin{gathered}
u^{\prime}(t)=M e^{\omega t} m(t) \Omega\left(\left|B^{-1}\right| \ell(t)\right) \\
\leq M e^{\omega t} m(t) \Omega\left(\left|B^{-1}\right| e^{\omega t} u(t)\right), 0 \leq t \leq b .
\end{gathered}
$$


From this, we obtain

$$
\begin{gathered}
\frac{1}{\left|B^{-1}\right|}\left[\left|B^{-1}\right| e^{\omega t} u(t)\right]^{\prime} \leq M m(t) \Omega\left(\left|B^{-1}\right| e^{\omega t} u(t)\right)+\frac{\omega}{\left|B^{-1}\right|}\left(\left|B^{-1}\right| e^{\omega t} u(t)\right) \\
\leq \hat{m}(t)\left[\Omega\left(\left|B^{-1}\right| e^{\omega t} u(t)\right)+\left|B^{-1}\right| e^{\omega t} u(t)\right]
\end{gathered}
$$

or

$$
\begin{aligned}
& \int_{\left|B^{-1}\right| u(0)}^{\left|B^{-1}\right| e^{\omega t} u(t)} \frac{d s}{s+\Omega(s)} \leq\left|B^{-1}\right| \int_{0}^{b} \widehat{m}(s) d s \\
& <\int_{c}^{\infty} \frac{d s}{s+\Omega(s)}, 0 \leq t \leq b .
\end{aligned}
$$

Therefore, we have

and consequently,

$$
\|y\|_{b} \leq \widehat{K}
$$

$$
\|x\|_{b} \leq K
$$

where $K$ is a constant, independent of $\lambda$, and the result follows.

Remark 5.2: In the special case, as in [8], where $m=L$ and $\Omega(t)=t+k$ we find

$$
K=|B|\left\{M|B|\|\phi\|+M L k \omega^{-1}\right\} \exp \left[\left(M L\left|B^{-1}\right|+\omega\right) b\right] .
$$

\section{Acknowledgements}

The authors are grateful to the referee(s) for his useful suggestions which improved the initial version of the paper.

\section{References}

[1] Dugundji, J. and Granas, A., Fixed Point Theory, Vol. I, Monographie Matematyczene, PNW, Warsawa 1982.

[2] Fitzgibbon, W., Semilinear functional differential equations in Banach space, $J$. Diff. Equations 29 (1978), 1-14.

[3] Friedman, A., Partial Differential Equations, Holt, Rinehart and Winston, New York 1969.

[4] Granas, A., Guenther, R. and Lee, J., Nonlinear Boundary Value Problems for Ordinary Differential Equations, Dissertationes Mathematicae, Warsaw 1985.

[5] Hale, J., Theory of Functional Differential Equations, Springer-Verlag, New York 1977.

[6] Kaminogo, T., Spectral approach to boundary value problems for functional differential inclusions, Funckcial. Ekvac. 27 (1984), 147-156. 
[7] Lee, J. and O'Regan, D., Topological transversality. Applications to initial value problems, Ann. Pol. Math. 48 (1988), 247-252.

[8] Lightbourne III, J. and Rankin III, S., A partial functional differential equation of Sobolev type, J. Math. Anal. Appl. 93 (1983), 328-337.

[9] Ntouyas, S., Sficas, Y. and Tsamatos, P., Existence results for initial value problems for neutral functional differential equations, J. Diff. Equations 114 (1994), 527-537.

[10] Rankin III, S., Boundary value problems for partial functional differential equations, J. Math. Anal. Appl. 90 (1980), 459-468.

[11] Rankin III, S., Existence and asymptotic behavior of a functional differential equation in Banach space, J. Math. Anal. Appl. 88 (1982), 531-542.

[12] Travis, C. and Webb, G., Existence and stability for partial functional differential equations, Trans. Amer. Math. Soc. 200 (1974), 395-418.

[13] Travis, C. and Webb, G., Existence, stability and compactness in the a-norm for partial differential equations. Trans. Amer. Math. Soc. 240 (1978), 129-143.

[14] Tsamatos, P. and Ntouyas, S., Existence of solutions of boundary value problems for differential equations with deviating arguments via the Topological Transversality method, Proc. Royal Soc. Edinburgh 118A (1991), 79-89.

[15] Ward, J., Boundary value problems for differential equations in Banach space, J. Math. Anal. Appl. 70 (1979), 589-598.

[16] Yorke, J., A continuous differential equation in Hilbert space without existence, Funkcial. Ekvac. 13 (1970), 19-21. 


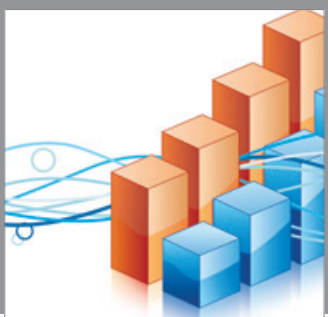

Advances in

Operations Research

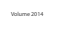

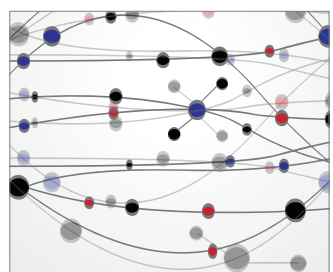

\section{The Scientific} World Journal
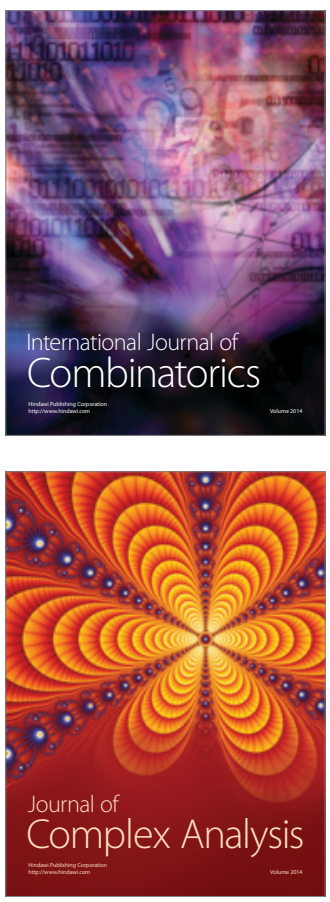

International Journal of

Mathematics and

Mathematical

Sciences
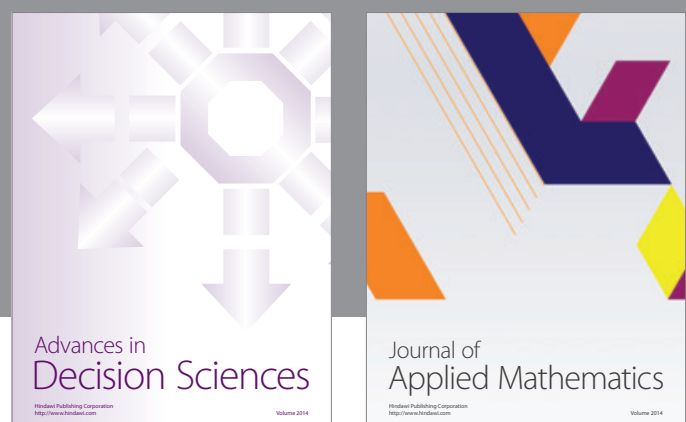

Journal of

Applied Mathematics
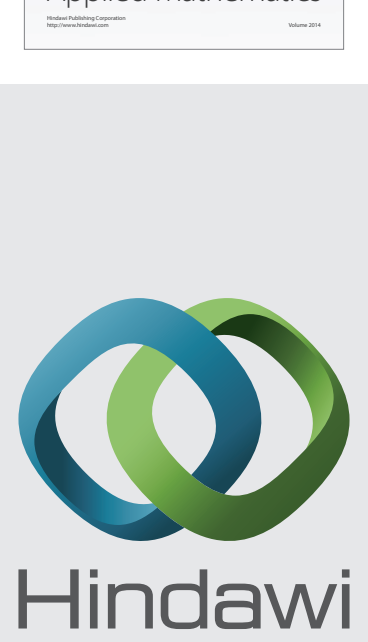

Submit your manuscripts at http://www.hindawi.com
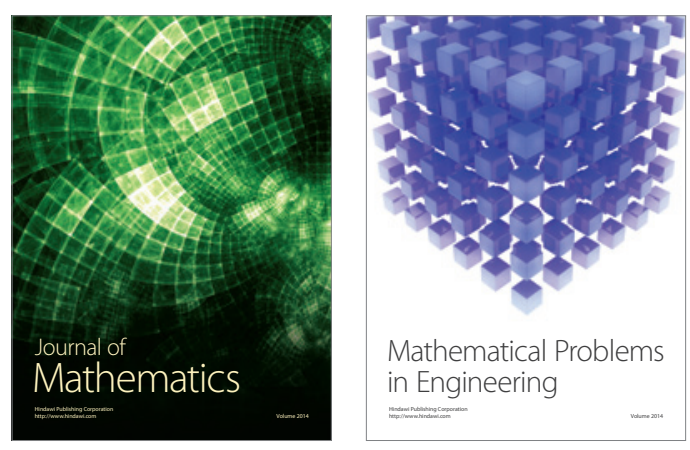

Mathematical Problems in Engineering
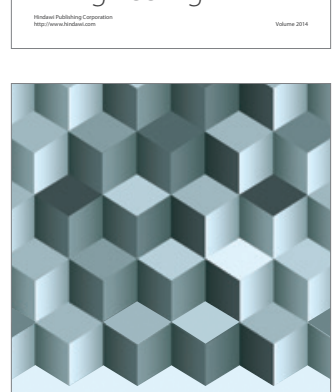

Journal of

Function Spaces
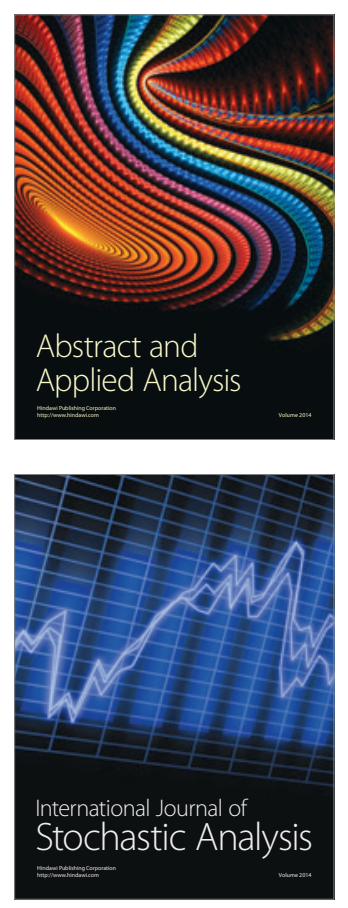

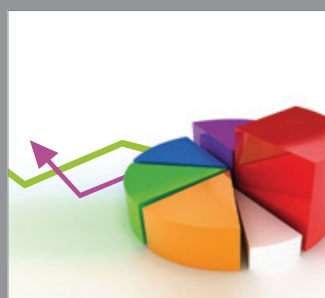

ournal of

Probability and Statistics

Promensencen
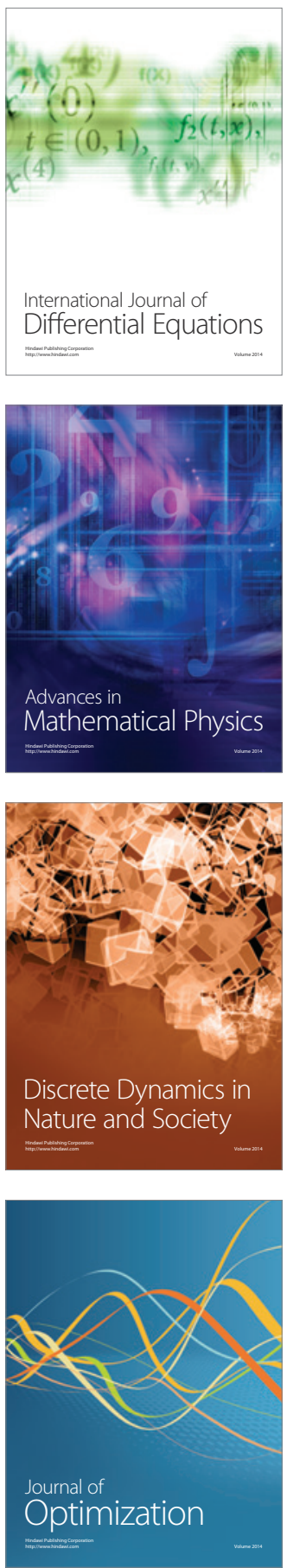